\title{
PROCESSING OF OPERATING DEFLECTION SHAPES
}

\section{PAVEL SURANEK, JIRI CZEBE, VACLAV PAVELKA, RENATA WAGNEROVA}

Dep. of Control Systems ans Instrumentation, Faculty of Mechanical Engineering, VSB-Technical University of Ostrava, Ostrava, Czech Republic

DOI : 10.17973/MMSJ.2019_03_201899

pavel.suranek@vsb.cz

The article deals with measurement and visualization of the operating deflection shapes (ODS) on laboratory task. The aim is to measure Frequency Response Function (FRF) of selected points of the template. Together with visualization of their magnitude, real part, imaginary part or phase and show template's ODS. The measuring chain consists of an accelerometer, laser and shaker. Data acquisition is performed via PULSE LabShop application. Visualization of processed data is accomplished in Matlab GUI application called "ODS app". The article also contains the theoretical basis of the measurement principle along with setup of PULSE LabShop project and general description of Matlab GUI application components.

KEYWORDS

Auto-spectrum, cross-spectrum, deflection shapes, frequency transfer function, Matlab, transfer function

\section{INTRODUCTION}

Operating deflection shapes (ODS) analysis is a method for visualization the object dynamic under self-exciting forces. This method enables to identify, where the machine is moving with the maximum displacement, velocity or acceleration and which frequencies of excitation cause these problems. It is useful tool for the diagnostics of faults and for the development of new components or whole machines.

Method is based on experimental measuring of frequency response functions and interpreting them as an animation of a simplified geometric model representing the examined sample. The principle of this method is divided into two phases. The first phase represents measuring of FRF, i.e. determination of vibration magnitudes at single characteristic points and mutual phases (usually to one reference signal). The next step is the creation of an animation, which illustrates the shape of vibration during the excitation by selected frequency or by the combination of more frequencies. The movement of structure is usually amplified and slowed-down in the visualisation to make it suitable for visual inspection.

We can rank the most important pros: simplicity, great information value and wide range of implementations. The method allows e.g. identification of dynamic properties (resonance frequencies, identification of largest displacement location on part and design of flexible fitting, misery or unbalance).

This method grew up on its importance at the end of 80's in the 20th century due to the increasing computing power and the implementation of two-channel (and later multi-channel) signal analysers into the diagnostics.

The operating deflection shapes analysis has become unnecessary part of machine diagnostics knowledge and this paper presents a laboratory task which is used for the education of mechanical engineers. The next chapter deals with a measurement of frequency response functions. It continues with the part which presents the relationship between frequency response functions and shapes, then an Matlab application for shape visualisation is introduced and finally, some results of the experiment are presented.

\section{FREQUENCY RESPONSE FUNCTION}

Frequency response function (FRF) represents the relation of the output and the input signal in a frequency domain. FRFs between measured points pairs are necessary for the ODS visualization [Herfert 2015], [Yonghui 2017], [Ganeriwala 2008]. It is accomplished in several steps. Two discretized signals are measured at the beginning. Let's call the output channel of the system simply as a "signal" and the input as a "reference signal". The signal is marked as $y(k T)$ and the reference signal as $x(k T)$. Both signals are then divided into data blocks with $2 / 3$ overlay and $M$ realizations of signals $y_{1}(k T) \ldots y_{M}(k T), x_{1}(k T) \ldots$ $\mathrm{x}_{M}(k T)$ are extracted. All the realizations have $N$ number of samples. In the next step, each of the blocks are weighted by Hanning window and obtained results are transformed using the Fast Fourier transform (FFT) into the frequency domain representations $Y_{1}(f) \ldots Y_{M}(f), X_{1}(f) \ldots X_{M}(f)$. Complex conjugates are calculated by changing the sign of the imaginary part of complex numbers and these representations are marked with superscript "*": $Y_{1}^{*}(f) \ldots Y_{M}^{*}(f), X_{1}^{*}(f) \ldots X_{M}^{*}(f)$. Now, it is possible to calculate auto-spectrum $S_{y y}$ of the signal, autospectrum $S_{x x}$ of a reference signal. And a cross spectrum $S_{x y}$.
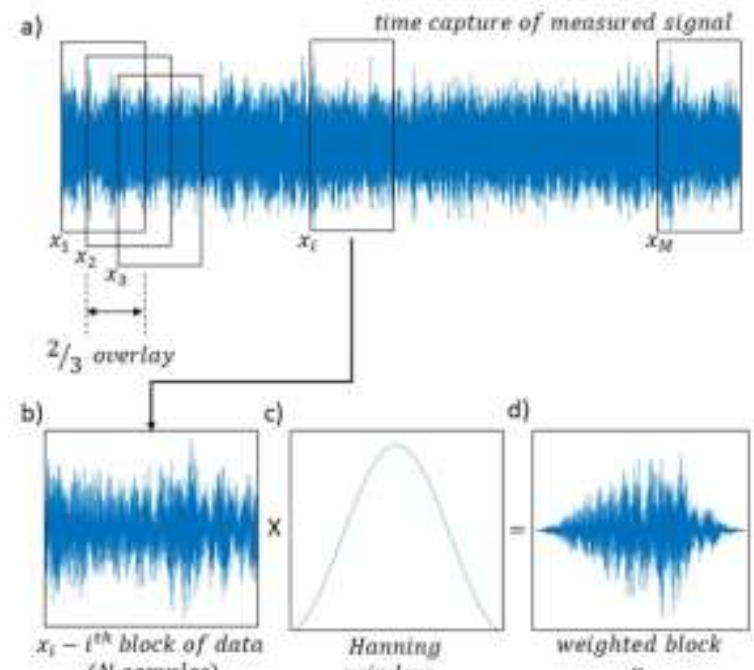
c) d)

Figure 1. Dividing the measured signal into $M$ blocks and weighting by Hanning window

The auto-spectrum (also known as power spectrum) of the signal weighted block is computed as a product of signal's Fast Fourier transform and corresponding complex conjugate. The resulting auto-spectrum is computed as an average over $M$ measurement realizations.

$$
S_{y y}(f)=\frac{1}{M} \sum_{i=1}^{M} Y_{i}(f) \cdot Y_{i}^{*}(f)
$$

The averaging smooths the resulting spectrum but also consumes measurement time, because more samples are needed.

The auto-spectrum of the reference signal is calculated in the same way:

$$
S_{x x}(f)=\frac{1}{M} \sum_{i=1}^{M} X_{i}(f) \cdot X_{i}^{*}(f)
$$


The cross-spectrum is computed as a multiplication between the Fourier transform of the reference signal and the corresponding complex conjugate of signal's Fourier transform.

$$
S_{x y}(f)=\frac{1}{M} \sum_{i=1}^{M} X_{i}(f) \cdot Y_{i}^{*}(f)
$$

It is also possible to calculate the "backwards" cross-spectrum:

$$
S_{y x}(f)=\frac{1}{M} \sum_{i=1}^{M} Y_{i}(f) \cdot X_{i}^{*}(f)
$$

Frequency response function is simply the cross-spectrum divided by the auto-spectrum of the reference signal:

$$
H 1(f)=\frac{S_{x y}(f)}{S_{x x}(f)}
$$

Alternatively, it is possible to calculate the FRF as the signal auto-spectrum divided by the cross-spectrum:

$$
H 2(f)=\frac{S_{y y}(f)}{S_{y x}(f)}
$$

If the frequency response function is calculated as $\mathrm{H} 1$ approach, the processing can suppress the noise of output signal $\mathrm{y}$ and if the function is calculated in a $\mathrm{H} 2$ way, the noise in the input signal is supressed. In the case, when it is supposed that both signals are affected by the noise on a comparable level, the "H3" is computed as a geometrical average of $\mathrm{H} 1$ and $\mathrm{H} 2$.

$$
H 3(f)=\sqrt{H 1(f) \cdot H 2(f)}
$$

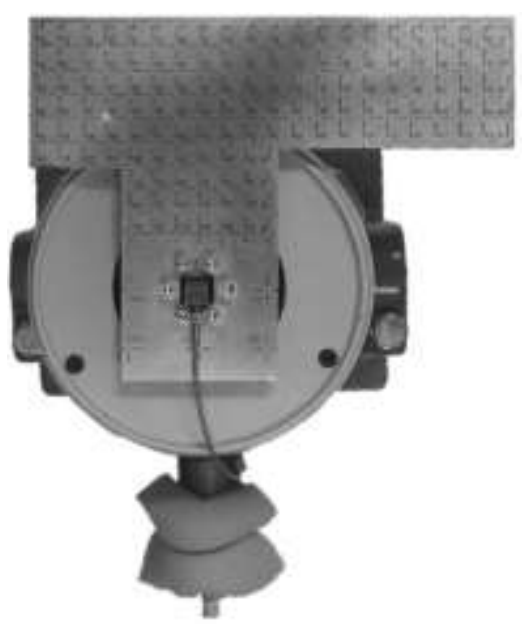

Figure 2. Sample's mesh with attached accelerometer and laser located at the selected measurement point.

The first step is to draw a mesh on a studied object. In this demonstration task, the number of 164 measured points is defined on the sample, the positions of the points reflect the sample's geometry. Using 6 bolts, the sample was fastened to the shaker and an accelerometer was glued onto the appropriate point in the center of this attachment. This accelerometer measures the reference signal and remains in the same position during all of 164 frequency response functions measurements. The output signal was measured using the laser vibrometer. The both signals are processed in the signal analyser BK $3560 \mathrm{C}$ and Pulse LabShop software.
Because the signal from the laser is delayed by $1,28 \mathrm{~ms}$, this time must be added also to the accelerometer channel (good synchronization is a key preposition of this kind of measurement). The analyser also generates a white noise signal for the structure's excitation. This signal is sent to the amplifier and the shaker's coil is powered.

White noise is a commonly used measurement signal, which has theoretically infinite frequency range. Practically it must be reduced to some finite range, in the measurement presented in this paper, the frequency span was $12.8 \mathrm{kHz}$. Another testing signals can also be used to identify various systems. The simplest one is sine testing signal, another are: swept sine, impulse, step, spread spectrum signal [Vala 2016], [Proto 2016].

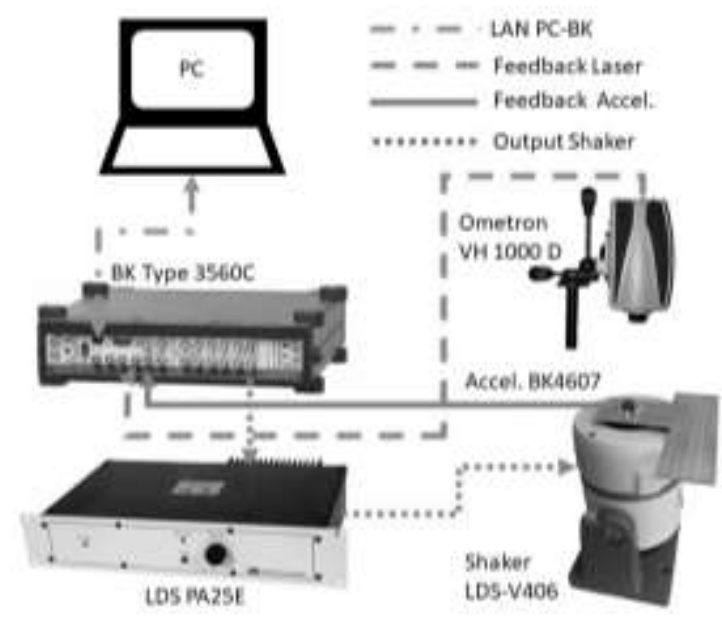

Figure 3. Measurement chain

Frequency response functions were measured in the range of $6.4 \mathrm{kHz}$. Sampling frequency is 2.56 times greater, which is 16384 samples per second. This satisfies the Nyquist-Shannon sampling theorem with a suitable reserve. The power of two number is eligible for Fast Fourier transform used later. The chart consisted of 1600 bars, which means that the block were 4096 samples long (2.56 times more); it corresponds to $250 \mathrm{~ms}$. FRFs were computed as an average from 100 blocks.

In next figures, there are examples of spectra and FRF, which were obtained during the measurement of point number 88 . Auto-spectra (Fig. 4) express which frequencies are present in the signal. Several frequencies in laser signal are dominant; it can be said, that these are the frequencies at which the sample tends to vibrate. Auto-spectra are real numbers, while the cross-spectrum is a sort of complex numbers and brings the information of the phase (Fig. 5) FRF includes two information because these functions are complex. Several approaches how to graphically demonstrate this FRF exists, but probably most often the magnitude and the phase both depending on the frequency are depicted. In this case the magnitude part shows how many times the output's amplitude is greater than the input's amplitude at selected frequency. However, for the purpose od ODS, the imaginary part (Fig. 7) is the most important one and is used for the illustration of the shape.

FRFs are presented for the purpose of ODS visualization in this paper, but these can be found in many more applications such are modal analysis [Maia 1997], [Agneni 2004], [Agneni 2006] or is a part in the design in popular passive and active vibration control [Wrona 2016A], [Wrona 2016B], [Wrona 2016C]. 

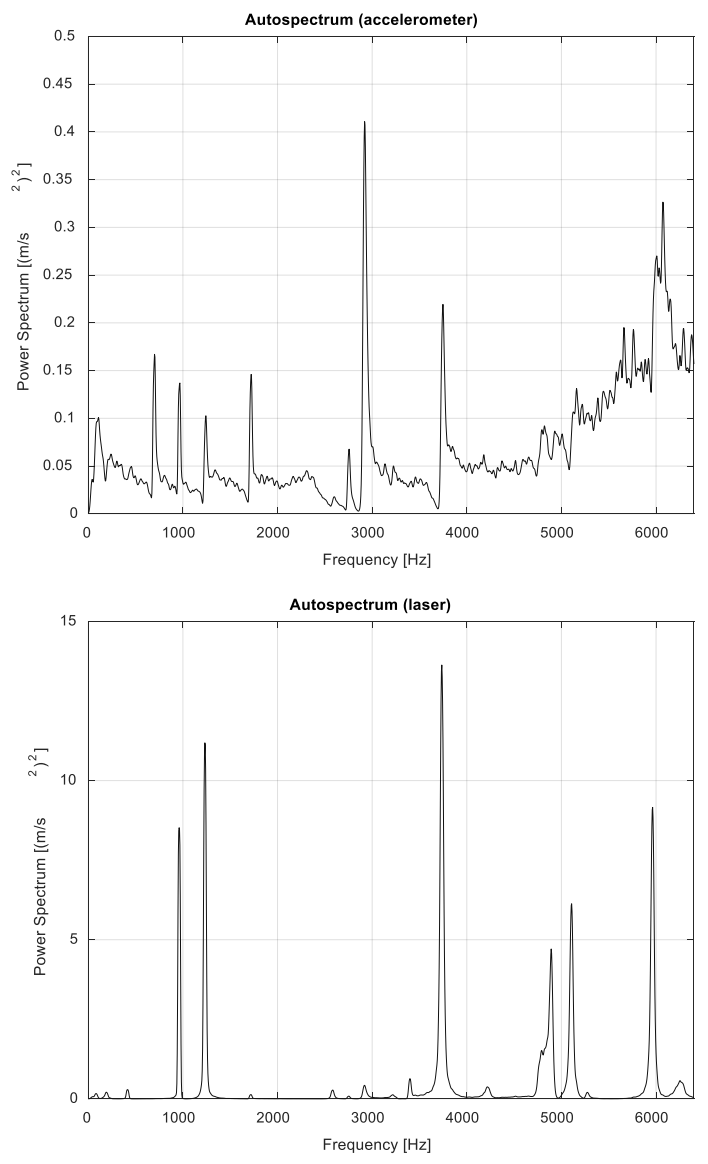

Figure 4. Auto-spectra of measured signals
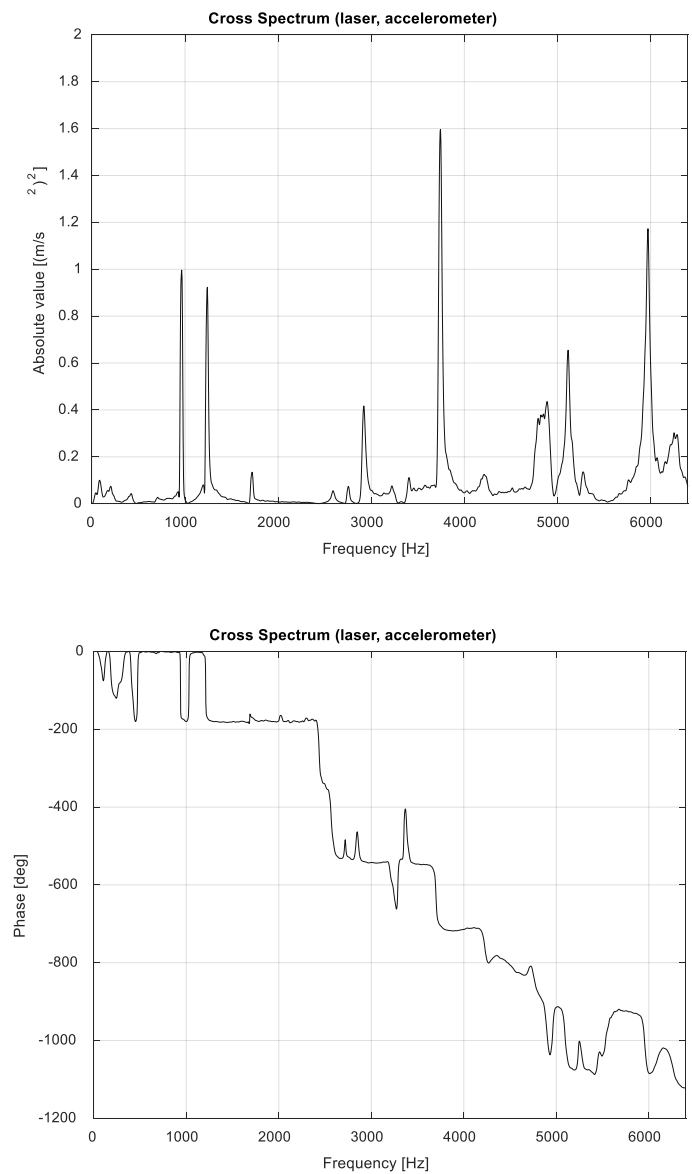

Figure 5. Cross-spectrum between measured signals
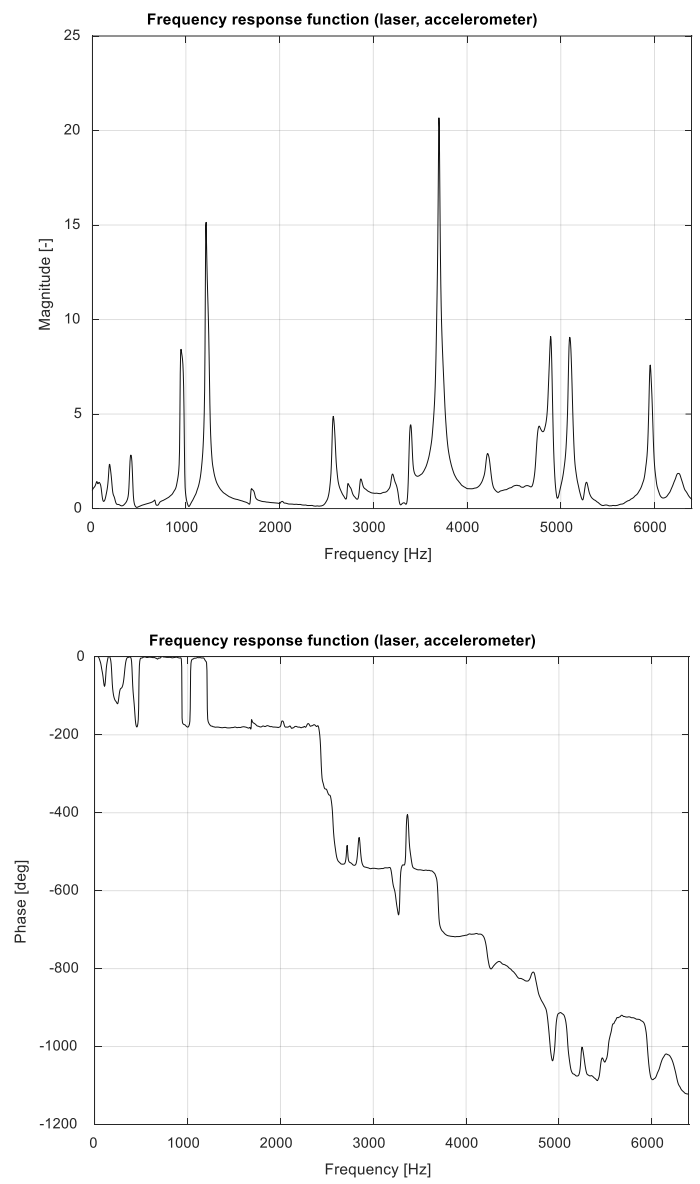

Figure 6. Frequency response function - magnitude and phase
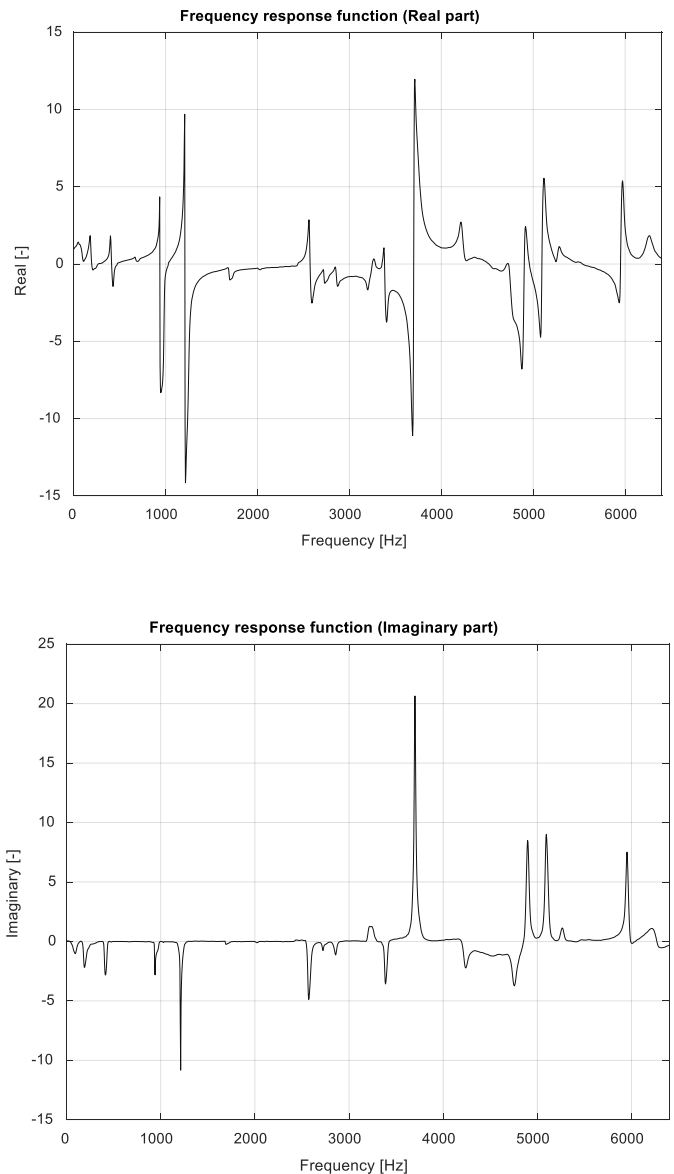

Figure 7. Frequency response function - real and imaginary part 


\section{DEFLECTION SHAPES}

In application presented in this paper, operating deflection shapes will be extracted from imaginary parts of measured response functions. The relationship between FRFs measured on a laboratory sample and final deflection shape is best to show on an example. Let's say, we want to study the deflection shape of the sample during the frequency of $1212 \mathrm{~Hz}$. We will focus on the measurement point number 88 , whose FRF was captured and described in the previous chapter. The value of imaginary part at the frequency of $1212 \mathrm{~Hz}$ is approximately 12. This number is placed into a mesh grid as a third value in a $3 \mathrm{D}$ surface plot. While this process is repeated for all measured points, the final shape for $1212 \mathrm{~Hz}$ is acquired.

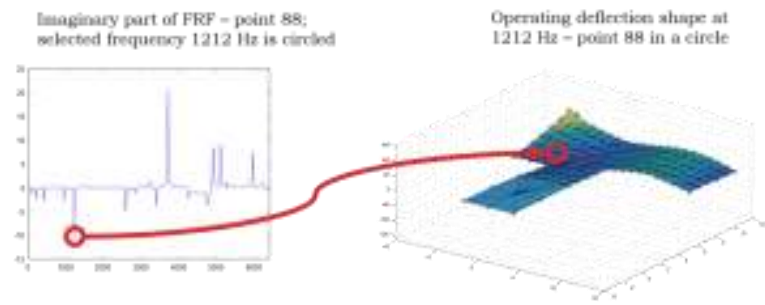

Figure 8. The relationship between measured FRF and the operating deflection shape

\section{ODS APPLICATION}

Graphical user interface was designed to visualize the measured data. This application enables to import measured FRFs saved as text files in ASCII format. These functions can be displayed as magnitude, phase, real and imaginary part. The selection of displayed functions is also implemented and is accessible through context menu. The main part of GUI is the visualization of the deflection shape according to the selected frequency. This shape can be animated, and the video can be saved in an avi file.

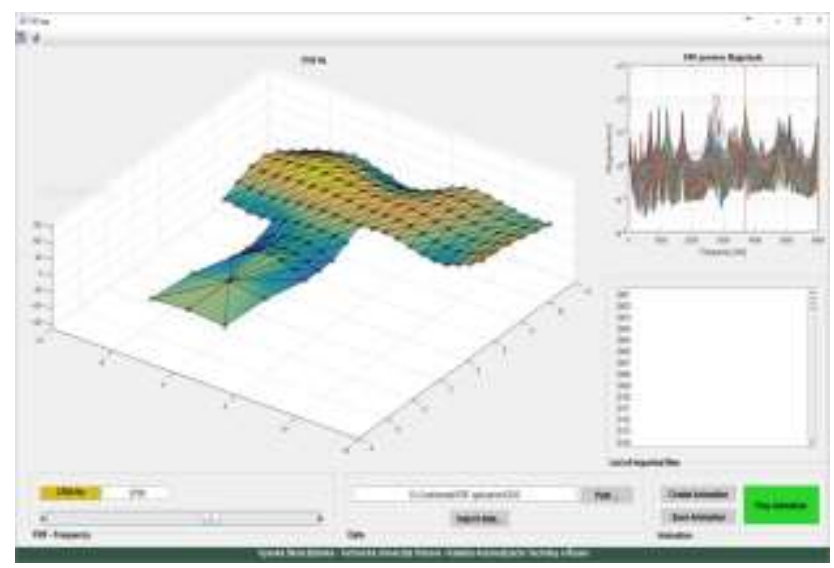

Figure 9. ODS application GUI preview

\section{EXPERIMENTAL RESULTS}

Results acquired on a laboratory plant are concluded in this chapter in a graphical form. Figures depict ODSs at a given frequency. These illustrations enable to understand which places on the laboratory samples have vibrationally risky behaviour and which frequencies are connected to these problems. For example, if we look at the Figure 12, which illustrates the shape at the frequency of $1220 \mathrm{~Hz}$, there can be seen, that the left corner, right edge and the area on the right side have the highest relative amplitudes.

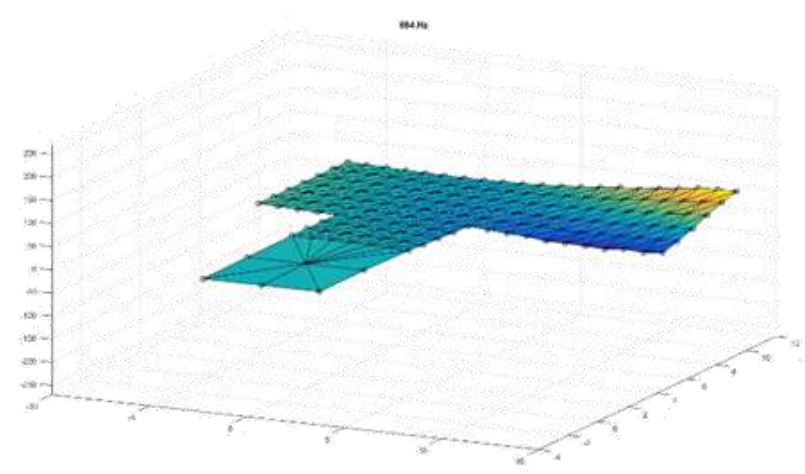

Figure 10. Shape at $684 \mathrm{~Hz}$

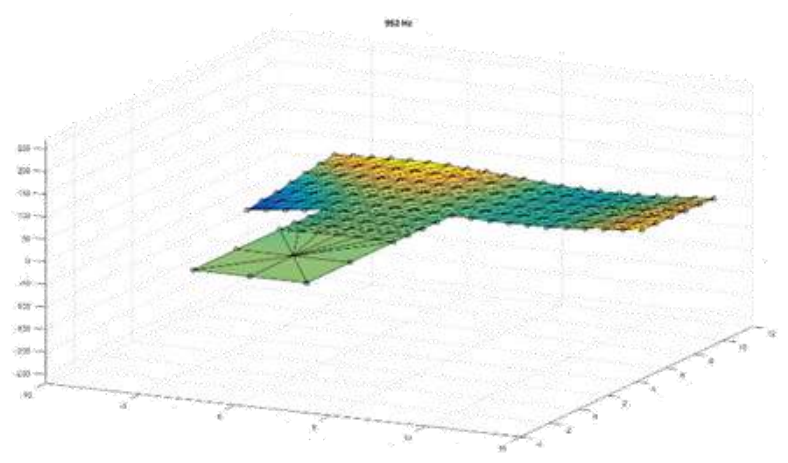

Figure 11. Shape at $952 \mathrm{~Hz}$

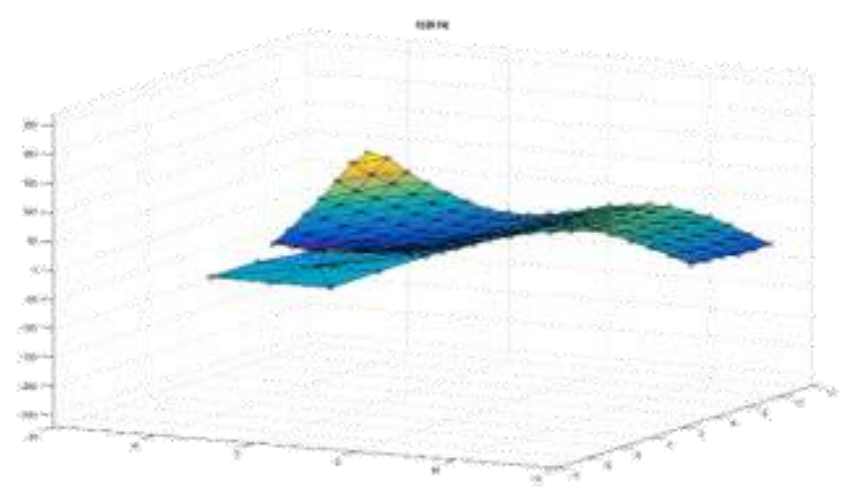

Figure 12. Shape at $1220 \mathrm{~Hz}$

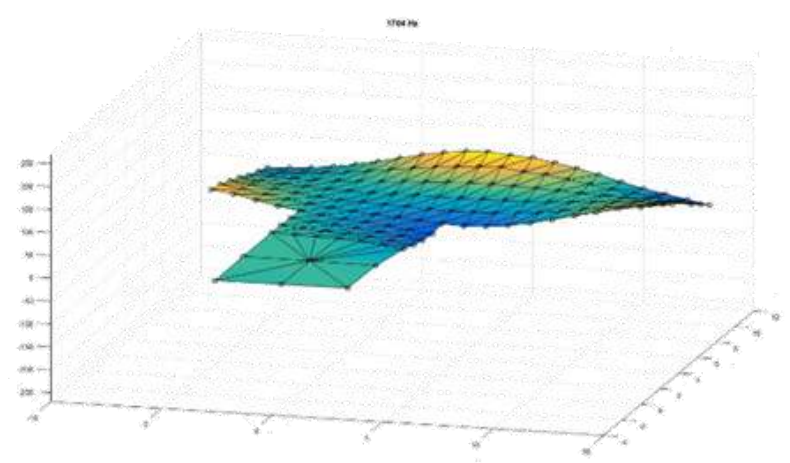

Figure 13. Shape at $1704 \mathrm{~Hz}$ 

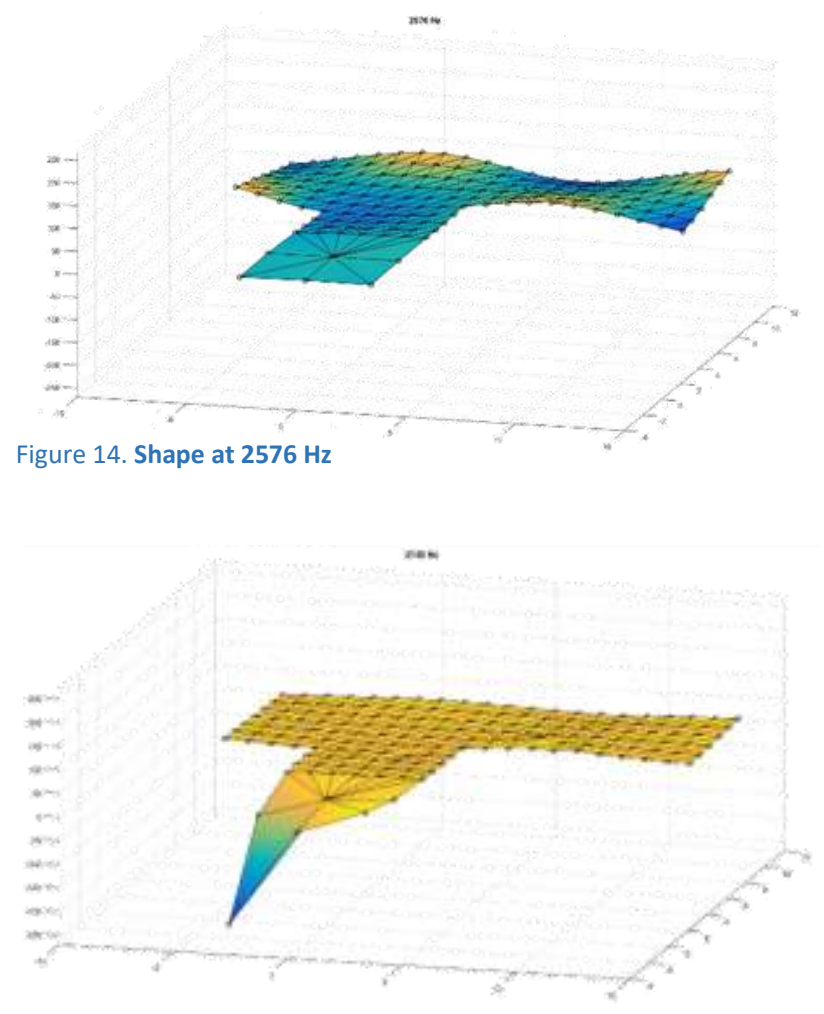

Figure 15. Shape at $2740 \mathrm{~Hz}$

\section{CONCLUSIONS}

This paper presents a laboratory task designed for operating deflection shape processing. The mathematical procedure used for measuring frequency response functions was introduced together with a measurement chain for the diagnostics of these functions. Laboratory equipment included a vibration shaker with its signal amplifier, signal analyser and signal processing software, piezoelectric accelerometer and Doppler laser vibrometer. The relation between frequency response functions and operating deflection shape was explained. A special application designed for the visualization of measured shapes was also presented and finally the experimental results obtained on a laboratory sample are shown.

The main goal of this paper is to simply demonstrate the acquiring operating deflection shapes in several simple steps: measurement FRFs on a laboratory sample, transferring imaginary parts onto a mesh and visualize results.

\section{ACKNOWLEDGMENTS}

This work was supported by the European Regional Development Fund in the Research Centre of Advanced Mechatronic Systems project, project number CZ.02.1.01/0.0/0.0/16_019/0000867 within the Operational Programme Research, Development and Education; and the project No: SP2019/51 „Applied Research in the Area of Machine and Process Control " supported by the Ministry of Education, Youth and Sports.

\section{REFERENCES}

[Agneni 2004] Agneni, A. et al. On modal parameter estimates from ambient vibration tests. In: Proceedings of the
2004 International Conference on Noise and Vibration Engineering, Leuven, September 20 -22, 2004. Leuven: Katholieke Universiteit Leuven, 22392248. ISBN 90-73802-82-2.

[Agneni 2006] Agneni, A. and Coppotelli, G. Load Spectrum Identification from Operative Responses. In: Proceedings of ISMA2006: International Conference on Noise and Vibration Engineering, Leuven, 18-20 September 2006, Leuven: Katholieke Universiteit Leuven, 3061-3069, 2016. ISBN 90-73802-83-0.

[Ganeriwala 2008] Ganeriwala, S. N. et al. Using Operating Deflection Shapes to Detect Misalignment in Rotating Equipment. In: Conference Proceedings of the Society for Experimental Mechanics Serie, Orlando February 4-7, 2008, 1-5.

[Herfert 2015] Herfert, D. and Gollnick, M. "Good Vibrations" A complete software solution for experimental, operational modal analysis and operating deflection shapes. In Proceedings of the 16th International Conference on Computer Systems and Technologies, Dublin June 2015, New York: ACM, pp. 46-53. ISBN 978-1-4503-3357-3

[Maia 1997] Maia, N. M. M., et al. Theoretical and Experimental Modal Analysis. Baldock, Hertfordshire: Research Studies Press, 1997

[Proto 2016] Proto, A., et al Measurements of generated energy/electrical quantities from locomotion activities using piezoelectric wearable sensors for body motion energy harvesting. Sensors, April 2016, vol. 16, no. 4, pp. 524. ISSN 1424-8220

[Vala 2016] Vala, D. Advanced AMR sensor using spread spectrum technology. IFAC-PapersOnLine, 2016, vol. 49, no. 25, pp. 511-516. ISSN 2405-8963

[Windalage 2009] Windalage, D. W., et al. Effects of Overlapping and Windowing on Frequency Response Function Estimates of Systems with Random Inputs. IEEE Transactions on Instrumentation and Measurement, 2009, vol. 58, no. 1 , pp. 214-220.

[Wrona 2016A] Wrona, S. and Pawelcyzk, M. Shaping frequency response of a vibrating plate for passive and active control applications by simultaneous optimization of arrangement of additional masses and ribs. Part I: Modeling. Mechanical Systems and Signal Processing, 2016, vol. 70-71, pp. 682-698. ISSN 0888-3270

[Wrona 2016B] Wrona, S. and Pawelcyzk, M. Shaping frequency response of a vibrating plate for passive and active control applications by simultaneous optimization of arrangement of additional masses and ribs. Part II: Optimization. Mechanical Systems and Signal Processing, 2016, vol. 70-71, pp. 699-713. ISSN 0888-3270

[Wrona 2016C] Wrona, S. and Pawelcyzk, M. Feedforward Control of a Light-Weight Device Casing for Active Noise Reduction. Archives of Acoustic, 2016, vol. 41, no. 3, pp. 499-505.

[Yonghui 2017] Yonghui, Y. Y., et al. Online Continuous Measurement of the Operating Deflection Shape of Power Transmission Belts Through Electrostatic Charge Sensing. IEEE Transactions on Instrumentation and Measurement, March 2017, vol. 66, no. 3 , pp. 492-501. 


\section{CONTACTS:}

Ing. Pavel Suranek, Ph.D.

VŠB - Technical University of Ostrava, Faculty of Mechanical Engineering, The Department of Control Systems and Instrumentation 17. listopadu 2172/15, Ostrava, 70800, Czech republic

+420 597324 113, pavel.suranek@vsb.cz, http://www.352.vsb.cz/ 